\title{
CONCORDANCIA ENTRE ECOCARDIOGRAFÍA PRENATAL Y POSNATAL EN PACIENTES CON CARDIOPATÍAS CONGÉNITAS: HOSPITAL DE NIÑOS DR. ROBERTO DEL RÍO
}

\author{
Paulo Valderrama E. ${ }^{1}$, Ignacio Hernández N. ${ }^{2}$ \\ ${ }^{1}$ Programa en Pediatría, Facultad de Medicina, Universidad de Chile. ${ }^{2}$ Servicio de Cardiología y Cirugía Cardiovascular, \\ Hospital de Niños, Dr Roberto del Río.
}

\section{RESUMEN}

Antecedentes: En Chile las malformaciones congénitas son la primera causa de mortalidad neonatal pre$\mathrm{coz}$, siendo las cardiopatías congénitas su principal factor. La incorporación de la ecocardiografía prenatal es de gran ayuda en su pesquisa precoz. Objetivos: Evaluar la ecocardiografía prenatal como prueba diagnóstica y su concordancia con la ecocardiografía posnatal. Métodos: Se analizaron embarazadas derivadas al Hospital de Niños Dr. Roberto del Río entre abril 2004 y abril 2008, por sospecha de cardiopatías congénitas con posterior control neonatal. Resultados: Se evaluaron 188 embarazadas tanto con diagnóstico de cardiopatía congénita como con ecocardiografía normal. La edad gestacional promedio de derivación fue de 32 semanas, siendo la principal causa la sospecha de cardiopatía congénita en el examen obstétrico de rutina. Como prueba diagnóstica se obtuvo una sensibilidad de $100 \%$ y especificidad de $40 \%$ con una moderada concordancia entre ésta y el examen postnatal. Como prueba diagnóstica la ecocardiografía prenatal, se obtuvo una sensibilidad de $100 \%$ y especificidad de $89 \%$ del examen, al ser evaluada como cardiopatías en general, con una muy buena concordancia entre ésta y el examen postnatal. Discusión: Existe una tardía derivación y diagnóstico prenatal de las cardiopatías congénitas a nivel nacional, siendo importante capacitar aún más a los obstetras que realizan este tamizaje. La ecocardiografía prenatal realizada en nuestro centro por cardiólogos es una confiable herramienta diagnóstica con una muy buena concordancia con la ecocardiografía posnatal.

\section{PALABRAS CLAVES: Cardiopatía congénita, ecocardiografía prenatal, prueba diagnóstica, índice kappa}

\section{SUMMARY}

Background: In Chile, congenital malformations are the first cause of early neonatal mortality, the congenital heart defects its the main factor. The incorporation of antenatal echocardiography is very helpful in your research early. Objectives: To evaluate prenatal echocardiography as a diagnosis test and their concordance with postnatal echocardiography. Methods: We analyzed pregnancy who were derived to the Hospital de Niños Dr. Roberto del Rio, between April 2004 and April 2008, where studied because suspicion of having congenital heart defects with subsequent neonatal control. Results: 188 pregnancy were evaluated either with a diagnosis of congenital heart defect or with normal echocardiography. The mean gestational age of derivation was 32 weeks, the main causes the suspicion of congenital heart defect on routine obstetric examination. When being studied as diagnosis test it was obtained a sensitivity of $100 \%$ and a specificity of $40 \%$ with a moderate concordance between both echocardiographies. When analyzing with the diagnosis test prenatal echocardiography, it was obtained a sensitivity of $100 \%$ and a specificity of $89 \%$ of the review, 
when evaluated as general heart defect, with a very good concordance between it and the postnatal examination. Discussion: There is a late referral and prenatal diagnosis of congenital heart defect at national level, being important to train obstetricians who perform this screening. Prenatal echocardiography performed by cardiologists at our center is a reliable diagnosis tool with a very good concordance with postnatal echocardiography.

\section{KEY WORDS: Congenital heart defect, prenatal echocardiography, diagnosis test, kappa index}

\section{INTRODUCCIÓN}

Las malformaciones congénitas son la primera causa de mortalidad neonatal precoz $(40 \%)$ y la segunda en la mortalidad infantil (32\%) (1). Dentro de ellas, las cardiopatías congénitas son la principal causa de mortalidad en el periodo neonatal precoz. En Chile en el año 2007, la mortalidad infantil fue 8,3 por 1000 nacidos vivos (nv) y la mortalidad perinatal 8,8 por $1000 \mathrm{nv}$, con sus componentes, la mortalidad fetal tardía de 4,5 por 1000 nv y la mortalidad neonatal precoz de 4,3 por 1000 nv.

Las cardiopatías congénitas son producidas por alteraciones en la organogénesis del corazón, desconociéndose en la mayoría de los casos los factores causales y sólo identificándose en el 10\% un agente etiológico (2), por lo que la mayoría de las alteraciones cardíacas son observadas en población sin factores de riesgo (3).

$\mathrm{La}$ incidencia de las cardiopatías congénitas es 8 por 1000 nv, constante a nivel mundial e independiente de factores como raza, condición socioeconómica o situación geográfica (2), con igual proporción para las cardiopatías congénitas mayores y menores. Esta incidencia subestima el valor real de cardiopatías en fetos, dado que muchos de estos terminan en abortos espontáneos o mortinatos, reflejando cardiopatías congénitas complejas o alteraciones cromosómicas asociadas con defectos cardíacos (4), estimándose la incidencia antenatal a más del doble de la reportada clásicamente en recién nacidos (5).

Después de la incorporación de la ecocardiografía prenatal en los últimos 30 años, han aparecido numerosos reportes extranjeros que evalúan su efectividad en la reducción de la mortalidad neonatal precoz. Algunos autores desestiman totalmente su utilidad (6-8), mencionando que realmente no reduce la mortalidad neonatal, mientras otros son más cautelosos en referir, que esto podría deberse a que las principales cardiopatías diagnosticadas en el periodo antenatal son las más severas o están asociada a otras anormalidades extracardiacas (9), no importando, por lo tanto, el periodo del diagnóstico ecocardiográfico.
Otros estudios mencionan, que dado que algunos países poseen una distribución geográfica en la cual puede realizarse una rápida derivación del paciente a centros especializados, el impacto real del diagnóstico prenatal podría verse disminuido (10). Sin embargo, otros reportes, demuestran la ventaja del diagnóstico antenatal en cardiopatías congénitas ductus dependiente (síndrome de hipoplasia de ventrículo izquierdo (7), transposición de grandes arterias (11) y coartación de la aorta (12), en los cuales se ha reportado un mejor pronóstico con el diagnóstico antenatal.

La sensibilidad del estudio ecocardiográfico prenatal posee gran variabilidad, con rangos que van de 0 a $80 \%$ de tasas de detección (13-15) identificándose algunos factores que afectan esta sensibilidad como la población estudiada, experiencia del operador, técnica de ecocardiografía, examen secuencial y sistemático, edad gestacional al momento del examen, peso materno, posición fetal y tipo del defecto cardíaco (16-17). Por otro lado, la especificidad posee valores bastante aceptables y homogéneos, que en la mayoría de los reporte es de $99 \%$.

Un diagnóstico prenatal preciso, permitirá un manejo obstétrico y perinatal adecuado, traduciéndose en un ahorro económico importante. Así el conocimiento antenatal de una malformación o genopatía incompatible con la vida, permitirá evitar la realización de conductas o procedimientos innecesarios y por otra parte, concentrar esfuerzos y recursos en aquellos recién nacidos que tienen posibilidad de sobrevida (18).

El objetivo de este estudio es evaluar la ecocardiografía prenatal como prueba diagnóstica y su concordancia con la ecocardiografía posnatal realizada en el Hospital de Niños, Dr. Roberto del Río.

\section{PACIENTES Y MÉTODOS}

Se seleccionaron a todas las embarazadas evaluadas durante el periodo comprendido entre abril 2004 y abril 2008 que fueron derivadas de centros obstétricos por probable cardiopatía congénita en sus fetos. De éstas, se analizaron sólo a aquellas 
embarazadas en quienes se les comprobó cardiopatía congénita, por el equipo de cardiología del Hospital de Niños Dr. Roberto del Río y que posteriormente fuera controlada dicha cardiopatía en el período posnatal con una ecocardiografía en los primeros 6 meses de vida, por el mismo equipo, o a través de una autopsia para definir el diagnóstico definitivo.

Se realizó además un estudio prospectivo de embarazadas cuyos fetos tenían diagnóstico ecocardiográfico prenatal normal para luego comprobar dicho diagnóstico a través de una ecocardiografía posnatal, en similar número al analizado para ecocardiografías con cardiopatías congénitas. Esto, dado que la mayoría de los fetos sin cardiopatía congénita no son controlados posteriormente en periodo de neonatal.

Todas las embarazadas y posteriormente neonatos fueron evaluados con el ecocardiógrafo General Electric Vivid 7. Se utilizó la visión de cuatro y cinco cámaras, eje largo, eje corto y arco aórtico empleando el modo M, bidimensional y Doppler color con transductor de 3.5 a $5.0 \mathrm{MHz}$ de frecuencia, para obtener una mejor resolución de la anatomía cardiaca fetal, en la medida que fuese factible realizar.

Se analiza datos de edad materna, edad gestacional y motivos de derivación.

Finalmente se obtiene la concordancia, con el método estadístico Índice Kappa, entre los diagnósticos de las ecocardiografías prenatales y posnatales y de esta última con los diagnósticos ecocardiográfico que motivaron la derivación. Se clasificaron en muy buena, buena, moderada, baja, insignificante y sin concordancia, de acuerdo a si los valores obtenidos están entre $1-0,8 ; 0,8-0,6$; $0,6-0,4 ; 0,4-0,2 ; 0,2-0$, y menor a 0 respectivamente.

Las cardiopatías congénitas fueron agrupadas en 4 niveles, de acuerdo a la clasificación de gravedad dada por el Plan AUGE de Chile 2010 (Tabla l) y analizadas en relación a estos niveles con la ecocardiografía posnatal.

\section{RESULTADOS}

Se estudiaron a 972 embarazadas desde el periodo de abril 2004 a abril 2008, de las cuales fueron finalmente analizadas 107 que cumplían tanto con el diagnóstico de cardiopatía congénita en sus fetos como con el posterior control ecocardiográfico en los primeros 6 meses de vida para definir el diagnóstico definitivo y 81 embarazadas con ecocardiografías fetales normales en un segundo estudio prospectivo.

La edad materna de ambos grupos fluctuaban entre 15 y 44 años, con un promedio de 29 años y una moda de 32 años. La edad gestacional promedio al momento del examen fue de 32 semanas (rango: 20-40 semanas). De los pacientes analizados por cardiopatía congénita, se encontraron registrados el motivo de derivación en $80 \%$ (86/107), siendo los factores de riesgo más frecuentes que determinaron la derivación la sospecha de cardiopatía congénita en el examen obstétrico de rutina $(76,7 \%)$, seguido en un porcentaje reducido, por la patología materna (Tabla II).

De los 66 casos derivados por sospecha de cardiopatía en el examen obstétrico de rutina, 49 de ellos tenía diagnóstico especifico de alguna cardiopatía congénita, el resto sólo tenía el diagnóstico general de cardiopatía congénita fetal. Al estudiar como prueba diagnóstica el examen obstétrico se obtiene que presenta una sensibilidad de $100 \%$ pero una baja especificidad de $40 \%$ IC $95 \%$ (-3 a $83 \%)$, con una moderada concordancia con la ecocardiografía posnatal, índice kappa observado 0,5449; IC95\% (0,0459 a 1).

Tabla I

\section{CLASIFICACIÓN DE CARDIOPATÍAS CONGÉNITAS SEGÚN PLAN AUGE 2010}

\begin{tabular}{cl}
\hline Nivel & Cardiopatías congénitas \\
\hline 1 & $\begin{array}{l}\text { Transposición de grandes arterias, tronco arterioso, hipoplasia de ventrículo izquierdo, hipoplasia y atresia } \\
\text { aórtica, atresia mitral, DVAP total obstructivo. }\end{array}$ \\
2 & $\begin{array}{l}\text { Tetralogía de fallot, hipoplasia de VD, atresia tricuspídea, atresia pulmonar, estenosis pulmonar moderada- } \\
\text { severa, enfermedad de Ebstein severa. }\end{array}$ \\
3 & CIA, CIV, seno venoso, DVAP total no obstructivo, DVAP parcial, canal auriculo-ventricular. \\
4 & CIA-OS, estenosis pulmonar leve, coartación aórtica, enfermedad de Ebstein leve.
\end{tabular}

DVAP: drenaje venoso anómalo pulmonar; VD: ventrículo derecho; CIA-OS: comunicación interauricular tipo ostium secundum; CIV: comunicación interventricular. 
Tabla II

MOTIVO DE DERIVACIÓN DE PACIENTES CON CARDIOPATÍAS CONGÉNITAS CON DIAGNÓSTICO PRENATAL

\begin{tabular}{lcc}
\hline Motivo de derivación & Casos & $\%$ \\
\hline Sospecha en examen obstétrico de rutina & 66 & 76,7 \\
Patología materna (diabetes mellitus pregestacional) & 6 & 6,9 \\
Genopatía & 5 & 5,8 \\
Antecedentes familiares de cardiopatía congénita & 5 & 5,8 \\
Consumo de medicamentos, drogas o infección durante embarazo & 4 & 4,6 \\
Total & 86 & 99,8 \\
\hline
\end{tabular}

Respecto a los pacientes analizados sin cardiopatías congénitas, se encontraron registrados $100 \%$ de los motivos de derivación, siendo los factores de riesgo más frecuentes que determinaron la derivación, la historia familiar de cardiopatías congénitas $(61,73 \%)$, seguido por la sospecha de cardiopatía congénita en el examen obstétrico de rutina $(19,75 \%)$ (Tabla III).

Al estudiar como prueba diagnóstica la ecocardiografía prenatal, utilizando como gold standard la ecocardiografía posnatal, se obtiene que presenta una sensibilidad de $100 \%$ y especificidad de $89 \%$ IC95\% (82 a 95\%) al ser evaluada como cardiopatías en general, sin embargo esta sensibilidad y especificidad varía de acuerdo a los niveles de gravedad clasificados por el Plan Auge. Se obtienen, además, valores predictivos y likelihood ratio de la ecocardiografía prenatal muy aceptables para una prueba diagnóstica (Tabla IV).

Al analizar la concordancia entre las ecocardiografías pre y posnatales, realizadas en el Hospital de Niños Dr. Roberto del Río, y tomando en cuenta a ésta última como gold standard, se obtiene que existe muy buena concordancia entre ambas ecocardiografías, al ser evaluadas tanto como cardiopatías en general, como según el nivel 3 , y una buena concordancia al ser comparadas según el nivel 4, 2 y 1 (Tabla IV).

\section{DISCUSIÓN}

En los pacientes controlados por el equipo de cardiología de nuestro hospital, se observa una edad materna y una edad gestacional promedio, al momento del examen ecocardiográfico, de 29 años y 32 semanas respectivamente, lo cual es un diagnóstico tardío si comparamos estos resultados con los estándares internacionales o guías norteamericanas publicadas, que estipulan que el tiempo óptimo para la visualización del corazón y tractos de salida es entre las 18 y 22 semanas de gestación (18). Este es un hecho relevante que se debe mejorar, ya que al considerar las características de nuestra geografía, un diagnóstico y derivación precoz puede cambiar el pronóstico del paciente al ser derivado oportunamente a un centro especializado en cardiopatías congénitas y así llegar eventualmente a disminuir la mortalidad neonatal precoz.

Tabla III

MOTIVO DE DERIVACIÓN DE PACIENTES SIN CARDIOPATÍAS CONGÉNITAS PRENATAL

\begin{tabular}{lcc}
\hline Motivo de derivación & Casos & $\%$ \\
\hline Historia familiar de cardiopatías congénitas & 50 & 61,73 \\
Sospecha de cardiopatía en examen obstétrico de rutina & 16 & 19,75 \\
Malformación extracardíacas & 7 & 8,6 \\
Patología materna: & 5 & 6,17 \\
$\quad$ Lupus eritematoso sistémico & 3 & 3,7 \\
$\quad$ Diabetes mellitus pregestacional & 2 & 2,47 \\
Medicamentos & 2 & 2,47 \\
Genopatía & 1 & 1,23 \\
Total & 81 & 99,95 \\
\hline
\end{tabular}


Tabla IV

EVALUACIÓN DE ECOCARDIOGRAFÍA PRENATAL COMO PRUEBA DIAGNÓSTICA Y SU CONCORDANCIA PARA CARDIOPATÍAS CONGÉNITAS

\begin{tabular}{lccccc}
\hline & General & Nivel tipo 4 & Nivel tipo 3 & Nivel tipo 2 & Nivel tipo 1 \\
\hline Prevalencia & 0,489 & 0,154 & 0,101 & 0,207 & 0,027 \\
Sensibilidad (\%) & 100 & 79 & 79 & 95 & 80 \\
IC95\% & & $65-94$ & $61-97$ & $88-100$ & $45-100$ \\
Especificidad (\%) & 89 & 97 & 99 & 89 & 98 \\
IC95\% & $82-95$ & $95-100$ & $98-100$ & $84-94$ & $97-100$ \\
Índice kappa & 0,88 & 0,79 & 0,84 & 0,74 & 0,65 \\
IC 95\% & $0,81-0,95$ & $0,66-0,91$ & $0,70-0,97$ & $0,63-0,85$ & $0,32-0,98$ \\
VPP (\%) & 89 & 85 & 94 & 70 & 57 \\
IC 95\% & $82-95$ & $72-99$ & $82-100$ & $57-82$ & $20-94$ \\
VPN (\%) & 100 & 96 & 98 & 99 & 99 \\
IC 95\% & & $93-99$ & $95-100$ & $96-100$ & $98-100$ \\
LR (+) & 8,73 & 31,53 & 133,42 & 8,83 & 48,8 \\
IC 95\% & $4,8-14$ & $11,7-84,4$ & $18,6-954,7$ & $5,5-14,1$ & $14,6-162,8$ \\
LR (-) & 0,0 & 0,21 & 0,21 & 0,06 & 0,2 \\
IC 95\% & & $0,1-0,43$ & $0,09-0,51$ & $0,01-0,22$ & $0,04-1,17$ \\
\hline
\end{tabular}

VPP: valor predictivo positivo; VPN: valor predictivo negativo; LR: likelihood ratio.

Resulta importante destacar que de todos los diagnósticos que motivaron la derivación a nuestro centro, la mayoría de los pacientes no presentaban factores de riesgo conocidos, sino que lo hicieron por la sospecha de cardiopatía congénita realizada por el examen obstétrico de rutina, lo que es coincidente con publicaciones nacionales e internacionales $(19,20)$. Al evaluar ésta ecocardiografía, presentó una sensibilidad de $100 \%$, por lo tanto se pudieron pesquisar todas las malformaciones cardiacas, pero una baja especificidad de $40 \%$ si la comparamos con la ecocardiografía posnatal realizada por un cardiólogo de nuestro hospital, como gold standard, con una concordancia moderada entre ambas ecocardiografías. Por lo tanto se puede concluir que es un muy buen método de tamizaje para cardiopatías fetales, pero se debe capacitar aún más a obstetras en esta materia para que exista una derivación más oportuna y así poder mejorar el pronóstico de éstas.

Al ser evaluada la ecocardiografía prenatal realizada por cardiólogos, se encontraron valores de sensibilidad y especificidad similares a los reportados en la literatura internacional, esto probablemente explicado por el estudio secuencial y sistemático que se realiza en nuestro hospital para evaluar la anatomía cardiaca fetal.

Se observo una muy buena concordancia entre las ecocardiografías prenatales y posnatales tanto en las cardiopatías en general como según el nivel 3 del Plan Auge y una buena concordancia al analizar los niveles 4, 2 y 1 de dicho plan con una adecuada sensibilidad y especificidad en estos niveles, no quedando claro el porque de éstas diferencias.

\section{CONCLUSIONES}

Al cumplir con los objetivos propuestos, podemos concluir que este trabajo es un aporte, tanto para obstetras quienes son los que derivan a las embarazadas, como para el equipo de cardiología del Hospital de Niños Dr. Roberto del Río, ya que nos permitió evaluar la forma en que estamos trabajando, en cuanto a los tiempos que evaluamos a nuestros pacientes que debe ser mejorado, como también la exactitud de nuestros diagnósticos ecocardiográficos y las conductas que tomamos a partir de éstos, ya sea para dar de alta, cuando la ecografía es normal, como para iniciar un manejo más precoz y oportuno, cuando encontramos una cardiopatía congénita diagnosticada antenatalmente. 


\section{BIBLIOGRAFÍA}

1. Instituto Nacional de Estadísticas. Anuarios de Demografía y de Estadísticas Vitales. Chile, 1991-2001.

2. Ministerio de Salud Pública de Chile. Guía clínica de cardiopatías congénitas operables en menores de 15 años. Primera edición. Santiago, 2005.

3. Sharland G. Routine fetal cardiac screening: what are we doing and what should we do? Prenat Diagn 2004;24:1123-9.

4. Viñals F, Giuliano A. Cardiopatías congénitas: Incidencia antenatal. Rev Chil Obstet Ginecol 2002;67:203-6.

5. Jaeggi F, Scholler G, Jones O, Cooper S. Comparative analysis of pattern, management and outcome of pre-versus postnatally diagnosed major congenital heart disease: a population-based study. Ultrasound Obstet Gynecol 2001;17:380-5.

6. Tworetzky W, McElhinney D, Reddy V, Brook M, Hanley $\mathrm{F}$, Silverman N. Improved surgical outcome after fetal diagnosis of hypoplastic left heart syndrome. Circulation 2001;103:1269-73.

7. Mahle W, Clancy R, McGaurn S, Goin J, Clark B. Impact of prenatal diagnosis on survival and early neurologic morbidity in neonates with the hypoplastic left heart syndrome. Pediatrics 2001;107:1277-82.

8. Yates R. The influence of prenatal diagnosis on outcome in patients with structural congenital heart disease. Prenatal Diagn 2004;24:1143-9.

9. Copel JA, Tan AS, Kleinman CS. Does a prenatal diagnosis of congenital heart disease alter short-term outcome? Ultrasound Obstet Gynecol 1997;10:237-41.

10. Bonnet D, Coltri A, Bureta G, Fermont L, Le Bidois $\mathrm{J}$, Kachaner J, et al. Detection of transposition of the great arteries in fetuses reduces neonatal morbidity and mortality. Circulation 1999;99:916-8.

11. Franklin O, Burch M, Manning N, Sleeman K, Gould $\mathrm{S}$, Archer N. Prenatal diagnosis of coartation of the aorta improves survival and reduces morbidity. Heart
2002;87:67-9.

12. Todros T, Faggiano F, Chiappa E, et al. Accuracy of routine ultrasonography in screening heart disease prenatally. Gruppo Piemontese for prenatal screening of congenital heart disease. Prenat Diagn 1997; 17:901-6.

13. Randall P, Brealey S, Hahn S, et al. Accuracy of fetal echocardiography in the routine detection of congenital heart disease among unselected and low risk populations: a systematic review. BJOG 2005;112:24-30.

14. Tegnander E, Williams W, Johansen OJ, et al. Prenatal detection of heart defects in a non-selected population of 30149 fetuses-detection rates and outcome. Ultrasound Obstet Gynecol 2006;27:252-65.

15. Tegnander E., Eik-Nes SH. The examiner's ultrasound experience has a significant impact on the detection rate of congenital heart defects at the second-trimester fetal examination. Ultrasound Obstet Gynecol 2006;28:8-14.

16. Wong SF, Chan FY, Cincotta RB, et al. Factors influencing the prenatal detection of structural congenital heart diseases. Ultrasound Obstet Gynecol 2003;21(1):19-25.

17. Rodríguez JG, Enríquez G, Hering E, et al. Centro de referencia perinatal oriente (CERPO): Evaluación de la experiencia del primer año. Rev Chil Obstet Ginecol 004;69:107-11.

18. Rychik J, Ayres N, Cuneo B, et al. American Society of Echocardiography guidelines and standards for performance of the fetal echocardiogram. J Am Soc Echocardiogr 2004;17:803-10.

19. Brick DH, Allan LD. Outcome of prenatally diagnosed congenital heart disease: an update. Pediatr Cardiology 2002;23:449-53.

20. Rodríguez JG, Doggenweiler F, Enríquez G, Hinrichsen M, Soler P, Alarcón R, et al. Diagnóstico prenatal de cardiopatías. Rev Chil Ultrasonografía 1999;2:40-7. 